\title{
Bladder cancer in women
}

\author{
Brian D Nicholson academic clinical fellow in primary care ${ }^{1}$, John S McGrath consultant urological \\ surgeon $^{2}$ honorary senior lecturer ${ }^{3}$, Willie Hamilton professor of primary care diagnostics ${ }^{3}$
}

${ }^{1}$ Department of Primary Health Care Services, Oxford University, Oxford OX2 6GG, UK; ${ }^{2}$ Royal Devon and Exeter NHS Foundation Trust, Exeter, UK; ${ }^{3}$ University of Exeter Medical School, Exeter EX2 4SG, UK

This is one of a series of occasional articles highlighting conditions that may be more common than many doctors realise or may be missed at first presentation. The series advisers are Anthony Harnden, university lecturer in general practice, Department of Primary Health Care, University of Oxford, and Richard Lehman, general practitioner, Banbury. To suggest a topic, please email us at practice@bmj.com

A 76 year old woman reports recurrent urinary frequency, dysuria, and malodorous urine. No bacterial growth has been identified on two midstream urine samples, though empirical treatment with antibiotics has improved her symptoms. After three months, an episode of visible haematuria prompts referral and a transitional cell carcinoma of the bladder is diagnosed.

\section{What are the types of bladder cancer?}

In developed countries $90 \%$ of bladder cancers are transitional cell carcinomas; squamous cell carcinomas (SCC) make up most of the remainder. ${ }^{12}$ In endemic areas, squamous cell carcinoma related to schistosomiasis accounts for $70 \%$ of cases. ${ }^{1}$ Around $20 \%$ are muscle invasive at diagnosis and are associated with a significantly poorer prognosis. ${ }^{3}$ The major risk factor is smoking, though chronic infection, radiotherapy, and (before regulation) industrial dyes have been implicated. ${ }^{24}$

\section{Why is it missed?}

Although bladder cancer is more common in men, women experience more delays: the English National Audit of Cancer Diagnosis in Primary Care (2009-10) estimated that annually 435 more women than men with bladder cancer experience a delay in diagnosis, but there are few data from UK primary care to explain this. ${ }^{6}$ With no effective screening tool for bladder cancer, it is usually diagnosed symptomatically, with haematuria being the most common presenting symptom for both sexes in primary care (likelihood ratio 59, 95\% confidence interval 51 to 57) ${ }^{37}$ The likelihood ratio summarises how many times more (or less) likely patients with bladder cancer are to have a particular feature than patients without bladder cancer. A ratio over 10 (or under 0.1 ) is considered to provide strong evidence to rule in (or rule out) bladder cancer.

A study of outpatient haematuria claims for 7649 patients aged $>65$ in the United States (female:male 1:2.43) also described a delay in diagnosis after presentation in women. The mean time to diagnosis was 85.5 days in women $(95 \%$ confidence interval 81.3 to 89.4 ) compared with 73.6 days (71.2 to 76.1 ) in men $(\mathrm{P}<0.001)$. This difference persisted over time; women were $26 \%$ more likely to experience delay at three months after the initial claim, $16 \%$ at six months, and $23 \%$ at nine months. ${ }^{8}$ During investigations, women undergo more urinalyses (1.39 $v 1.19, \mathrm{P}<0.001)$ and urine cultures $(0.83 v 0.53, \mathrm{P}<0.001)$ and receive more diagnoses of urinary tract infections (odds ratio $2.32,95 \%$ confidence interval 2.07 to $2.59 ; \mathrm{P}<0.001$ ) and more antibiotics $(40.1 \% \vee 35.4 \%, \mathrm{P}<0.001)$, yet are less likely to undergo bladder imaging (odds ratio 0.80, 0.71 to 0.89 ; $\mathrm{P}<0.001){ }^{8}$

Bladder cancer is also associated with voiding disorders and abdominal pain, but European data from primary care and gynaecology report that women with these complaints are more likely to be treated empirically, with treatments given without further evaluation in $47 \%$ of women compared with $19 \%$ of men in the year before diagnosis $(\mathrm{P}<0.05) .{ }^{9}$

Although these data are not wholly derived from primary care, it is implicit that prolonged investigation and treatment for urinary tract infections over repeated consultations (without confirmation of symptom resolution) is more of a problem in women.

\section{Why does this matter?}

Although there are recognised sex differences in tumour biology, bladder anatomy, and environmental and hormonal exposures that contribute to differential outcomes, there is some evidence of a correlation between delay in primary care and worsening prognosis. ${ }^{10-12}$ 


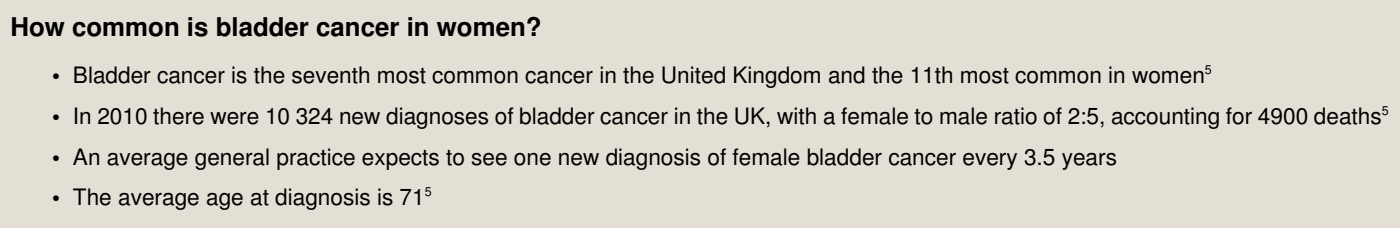

Prospective data from the UK for 1537 cases of bladder cancer (1340 detailing stage, and 633 both stage and cause of death) showed an association between longer delay between start of symptoms and GP referral and a 5\% increased incidence of muscle invasive bladder cancer (pT2-4) $(\mathrm{P}=0.04) .{ }^{12}$ Five year survival was significantly worse for women presenting with muscle invasive disease $(\mathrm{P}<0.001){ }^{12}$ While the report did not distinguish between patient delay and GP delay, longer delay before referral ( $>14 v<14$ days) resulted in an increased risk of death (hazard ratio $1.19,95 \%$ confidence interval 1.01 to 1.42 ; $\mathrm{P}=0.04)$ and $5 \%$ poorer five year survival $(\mathrm{P}=0.02) .{ }^{12}$ Patients with delay in their referral pathway seem to have more advanced disease, with poorer outcomes; arguably, they warrant the most expedient intervention compared with other groups.

\section{How is it diagnosed? Clinical features}

The National Institute for Health and Care Excellence (NICE) recommends urgent urology referral for adults with visible haematuria in the absence of urinary tract infection or in the presence of recurrent or persistent urinary tract infection in those aged $>40$; for unexplained microscopic haematuria in those aged $>50$; and for an abdominal mass arising from the bladder; and a non-urgent referral in those aged $<50$ with unexplained microscopic haematuria without raised creatinine concentration or proteinuria. ${ }^{13}$

Most studies in primary care have examined haematuria alone, though two-which both used pre-existing medical records-reported a broad range of symptoms associated with bladder cancer per $\mathrm{se}^{3}$ and with urinary tract cancer collectively. ${ }^{14}$ Most patients presented with painless haematuria, voiding symptoms, or a combination of the two.

\section{Haematuria}

The case-control study using electronic medical records from UK primary care showed that painless macroscopic haematuria is the strongest predictor of bladder cancer in primary care (odds ratio $34,95 \%$ confidence interval 29 to 41 ), with a positive predictive value in patients aged over 60 of $3.9 \%$ (3.5\% to $4.6 \%){ }^{3}$ The national audit showed that two thirds of patients had haematuria recorded as their primary symptom in primary care $^{6}$; though the prospective secondary care study showed that $90 \%$ of referred patients had haematuria (the severity of which did not correlate with disease severity); $25 \%$ of those referred will have transitional cell carcinoma of the bladder. ${ }^{6}{ }^{12}$

\section{Less specific symptoms}

The case-control study also showed that the commonly encountered symptoms of dysuria (odds ratio $4.1,95 \%$ confidence interval 3.4 to 5.0 ), abdominal pain (2.0, 1.6 to 2.4), and constipation (1.5, 1.2 to 1.9$)$, and a diagnosis of urinary tract infection $(2.2,2.0$ to 2.5$)$ are associated with bladder cancer but with much lower predictive values. ${ }^{3}$ Patients with more advanced disease might present with pelvic pain or urinary obstruction. In these patients, a mass might be palpable.
Importantly, re-attendance with persistent symptoms is associated with an increased risk of cancer.

\section{Investigations}

\section{Non-specific}

Urinalysis is accurate in detecting haematuria, proteinuria, nitrites, or leucocyte esterase, with subsequent microscopy and culture to confirm infection. Although three blood tests with abnormal results - raised white cell count, raised inflammatory markers, and raised creatinine - are associated with bladder cancer, these tests alone cannot be used to rule out the condition. ${ }^{3}$ The main role of urine cytology is in follow-up of patients with carcinoma in situ, rather than in the diagnosis of cancer. No primary care studies have reported its performance characteristics in diagnosis, but sensitivity is only $38 \%$ in secondary care and probably lower in primary care. ${ }^{15}$

\section{Definitive}

Flexible cystoscopy is the mainstay of investigation and is performed on a day case basis. It allows direct visualisation and biopsy of bladder abnormalities, but not treatment.

Ultrasongraphy of the renal tract is often added as the symptoms of bladder and renal cancer overlap. ${ }^{6}$ For patients with bladder cancer, staging might require computerised tomography and isotope bone scanning; positron emission tomography is increasingly used in specialist centres.

\section{How is it managed?}

Initial treatment depends on staging; less advanced disease is resected transurethrally (TURBT). Patients with low risk disease are offered cystoscopic surveillance, while recurrent low risk disease or intermediate/high risk disease can require intravesical chemotherapy or immunotherapy (with BCG). More advanced disease can require cystectomy or radical radiotherapy, with or without neo-adjuvant chemotherapy. ${ }^{2}$

Contributors: BDN performed the initial searches and drafted the first version of the article. All authors revised the article, adding additional references. WH is guarantor.

Competing interests: We have read and understood the BMJ policy on declaration of interests and declare the following interests: WH has had many research grants relating to cancer diagnostics from

non-commercial sources, including an NIHR programme grant which incorporates a bladder cancer study. He is the clinical lead for the current revision of the NICE 2005 guidance (CG 27) on the topic of selection of patients for cancer investigation. His contribution to this article is in a personal capacity and is not to be interpreted as representing the view of the Guideline Development Group or NICE. He has received travel support to give lectures and attend conferences, plus occasional speaker's fees, from conference organisers in the charitable and educational sector, though none from commercial sources. BDN is funded by the National Institute for Health Research (NIHR) School for Primary Care Research (SPCR). The views expressed are those of the author(s) and not necessarily those of the NHS, the NIHR, or the Department of Health. 


\section{Key points}

Women with bladder cancer are more likely to present with muscle invasive disease, partly associated with delays in diagnosis in primary care

Haematuria is the most highly predictive symptom of bladder cancer, and appropriate investigation and referral should not be delayed If women are treated for presumed urinary tract infection, active follow-up should ensure that symptoms have settled with treatment with antibiotics

Symptomatic women aged over 50 without confirmation of infection on urinalysis or microscopy and culture should be referred for further assessment to identify a definitive cause

Provenance and peer review: Not commissioned; externally peer reviewed.

Patient consent: Not required (hypothetical).

Kaufman DS, Shipley WU, Feldman AS. Bladder cancer. Lancet 2009;374:239-49. Lunt CR, Maddineni SB, Brough R. Bladder cancer. Br J Med Surg Urol 2012;5:95-103. Shephard EA, Stapley S, Neal RD, Rose P, Walter FM, Hamilton WT. Clinical features of bladder cancer in primary care. Br J Gen Pract 2012;62:e598-604.

4 Jemal A, Bray F, Center MM, Ferlay J, Ward E, Forman D. Global cancer statistics. CA Cancer J Clin 2011;61:69-90.

5 Bladder cancer. Cancer Research UK, 2013. www.cancerresearchuk.org/cancer-help/ type/bladder-cancer/

6 Lyratzopoulos G, Abel GA, McPhail S, Neal RD, Rubin GP. Gender inequalities in the promptness of diagnosis of bladder and renal cancer after symptomatic presentation: evidence from secondary analysis of an English primary care audit survey. BMJ Open 2013;3:e002861

7 Allaby M. Screening for bladder cancer using the urinalysis/dipstick method: a report for the national screening committee. NHS, 2010. www.screening.nhs.uk/policydb download. php?doc $=62$

8 Cohn JA, Vekhter B, Lyttle C, Steinberg GD, Large MC. Sex disparities in diagnosis of bladder cancer after initial presentation with hematuria. Cancer 2013;120:555-61.

9 Henning A, Wehrberger M, Madersbacher S, Pycha A, Martini T, Comploj E, et al. Do differences in clinical symptoms and referral patterns contribute to the gender gap in bladder cancer? BJU Int 2013;112:68-73.

10 Fajkovic H, Halpern JA, Cha EK, Bahadori A, Chromecki TF, Karakiewicz PI, et al. Impact of gender on bladder cancer incidence, staging, and prognosis. World J Urol 2011;29:457-63.

11 Shariat SF, Sfakianos JP, Droller MJ, Karakiewicz PI, Meryn S, Bochner BH. The effect of age and gender on bladder cancer: a critical review of the literature. BJU Int 2010;105:300-8.
12 Wallace DM, Bryan RT, Dunn JA, Begum G, Bathers S, on behalf of the West Midlands Urological Research Group. Delay and survival in bladder cancer. BJU Int 2002;89:868-78.

13 NICE. Referral for suspected cancer (CG27). National Institute for Health and Care Excellence, 2005.

14 Hippisley-Cox J, Coupland C. Identifying patients with suspected renal tract cancer in primary care: derivation and validation of an algorithm. Br J Gen Pract 2012;62:e251-60.

15 Planz B, Jochims E, Deix T, Caspers HP, Jakse G, Boecking A. The role of urinary cytology for detection of bladder cancer. Eur J Surg Oncol 2005;31:304-8.

Accepted: 5 February 2014

Cite this as: $B M J 2014 ; 348: g 2171$

\section{Related links bmj.com/archive}

Previous articles in this series

- Subdural haematoma in the elderly (BMJ 2014;348:g1682)

- Intestinal malrotation and volvulus in infants and children (BMJ 2013;347:f6949)

- Lisfranc injuries (BMJ 2013;347:f4561)

- Spontaneous oesophageal rupture (BMJ 2013;346:f3095)

- Pelvic inflammatory disease (BMJ 2013;346:f3189)

(c) BMJ Publishing Group Ltd 2014 